\title{
Влияние баллистической утечки на температурную зависимость квантового выхода светодиодов на основе множественных квантовых ям InGaN/GaN
}

\author{
(ㄱ И.А. Прудаев, В.В. Копьев, И.С. Романов, В.Л. Олейник
}

Томский государственный университет, 634050 Томск, Россия

E-mail: funcelab@gmail.com

(Получена 29 марта 2016 г. Принята к печати 7 апреля 2016 г.)

В работе исследованы зависимости квантового выхода от температуры и уровня возбуждения для светодиодов на основе множественных квантовых ям InGaN/GaN. Эксперимент проводился для двух режимов возбуждения люминесценции. Сравнение результатов, полученных при фото- и электролюминесценции, показало, что в области высокой плотности тока наблюдаются дополнительные низкотемпературные потери (дополнительные к потерям, связанным с оже-рекомбинацией). Это обусловливает инверсию температурной зависимости квантового выхода при температурах меньше $220-300 \mathrm{~K}$. В результате анализа установлено, что потери связаны с утечкой электронов из активной области светодиода. Для объяснения экспериментальных данных привлечена модель баллистической утечки. Результаты моделирования качественно согласуются с экспериментальными зависимостями квантового выхода от температуры и плотности тока.

DOI: 10.21883/FTP.2017.02.44112.8251

\section{1. Введение}

Температурная зависимость внешнего и внутреннего квантового выхода (КВ) светодиодов на основе множественных квантовых ям (МКЯ) InGaN/GaN ранее исследовалась в ряде работ, например [1-16]. Наиболее часто для исследования используются два метода: фотолюминесценция (ФЛ) и электролюминесценция (ЭЛ). Режим фотолюминесценции удобен тем, что позволяет частично, а в некоторых случаях и полностью исключить утечку носителей заряда из квантовых ям (КЯ). Так, например, в области криогенных температур, когда утечка носителей происходит по туннельному механизму, время жизни оказывается много меньше времени туннелирования [17], что позволяет сделать вывод о полном отсутствии утечки носителей в режиме ФЛ. При более высоких температурах роль утечки может быть выражена сильнее, если не выполняются стационарные условия возбуждения [18]. Тем не менее утечка носителей при ФЛ имеет меньшее значение, так как в данном режиме протекает биполярная генерация носителей, в отличие от электролюминесценции, когда активная область содержит большее количество электронов - носителей заряда с высокой подвижностью.

Вероятно, температурная зависимость КВ для структур с КЯ InGaN/GaN может быть строго описана с учетом температурной зависимости коэффициентов безызлучательной рекомбинации Шокли-Рида $(A)$, излучательной $(B)$ и оже-рекомбинации $(C)$ в рамках $A B C$-модели (см., например, для режима ФЛ [1-4]), хотя в литературе на этот счет до сих пор нет единого мнения $[19,20]$. В случае $A B C$-модели при всех уровнях возбуждения следует ожидать уменьшения КВ при росте температуры, что связано с ростом коэффициентов $A$ и $C$ [21]. Однако, несмотря на это, в некоторых работах авторы наблюдают инверсию температурной зависимости КВ для режима ФЛ: при росте температуры до $T=150-220 \mathrm{~K}$ квантовый выход увеличивается [5-7]. В области низкой интенсивности, когда имеют место два конкурирующих процесса, связанных с безызлучательной (Шокли-Рида) и излучательной рекомбинацией, рост КВ достигает относительно больших значений (по данным работы [7] до $\sim 250 \%)$. В области высоких уровней накачки, когда существенным становится темп оже-рекомбинации, рост КВ при увеличении $T$ относительно невысок (до $\sim 15 \%$ по данным работы [6]). В обоих случаях инверсия температурной зависимости КВ объясняется делокализацией носителей заряда с центров безызлучательной рекомбинации при повышении температуры [5-7].

В режиме электролюминесценции инверсия температурной зависимости КВ может приводить к более сильному росту квантового выхода с увеличением $T$ (от 1.5- до 30-кратного увеличения в области высоких плотностей тока, $j>(1-10) \mathrm{A} / \mathrm{cm}^{2}$ [8-14]). Для объяснения данного эффекта авторы различных работ рассматривали туннельные утечки [8], высокий уровень инжекции электронов в $p$-область $[9,10]$, выброс носителей из КЯ [11], активацию рекомбинационных центров при увеличении концентрации носителей [22], баллистический транспорт электронов $[12,13]$. Кроме того, рассматривались возможные причины инверсии температурной зависимости КВ для высокой плотности тока, связанные с изменением коэффициента $B$ при снижении $T$ вследствие изменения плотности состояний в валентной зоне [20]. Также стоит отметить, что рост КВ при снижении $T$ может наблюдаться в случае эффектов разогрева светодиода или стягивания тока [20,23], если некорректно выбраны условия измерений или конструкция экспериментальных образцов. Полный анализ воз- 
можных причин инверсии температурной зависимости КВ необходимо проводить с учетом конкретных условий эксперимента и структуры образцов.

В настоящей работе представлены экспериментальные результаты исследования температурной зависимости КВ в двух режимах (ФЛ и ЭЛ). Для анализа зависимости КВ от $j$ и $T$ привлекается одна из возможных моделей модель баллистической утечки носителей. Данная модель предложена ранее и использована для объяснения сложного вида вольт-амперных характеристик (BAX) светодиодов на основе МКЯ InGaN/GaN [13].

\section{2. Эксперимент}

В эксперименте использовалась коммерческая светодиодная структура „синего“ диапазона, выращенная методом газофазной эпитаксии из металлоорганических соединений на профилированной подложке $\mathrm{Al}_{2} \mathrm{O}_{3}$ (0001). Плотность прорастающих дислокаций, определенная по ямкам травления, не превышала $10^{8} \mathrm{~cm}^{-2}$. Общая толщина активной области структур составляла $\sim 200 \mathrm{Hм}$ и включала буферный слой, множественные квантовые ямы и барьерный слой $p-\mathrm{Al}_{0.15} \mathrm{Ga}_{0.85} \mathrm{~N}$ толщиной 30 нм. МКЯ $n$ - $\operatorname{In}_{0.15} \mathrm{Ga}_{0.85} \mathrm{~N} / \mathrm{GaN}$ имели период $\sim 17.5 \mathrm{Hм}$; по данным измерения фотопроводимости при резонансном возбуждении (405 нм) всего в активной области 7 КЯ. Толщина $p$-слоя, определенная методом атомно-эмиссионной спектроскопии, составляла $\sim 280$ нм. Из данных структур изготавливались светодиоды по планарной технологии со встречно-штыревыми омическими контактами $\mathrm{Ni} / \mathrm{Au}$ (контактное сопротивление, измеренное методом TLM (transmission line method), не превышало $\left.10^{-2} \mathrm{OM} \cdot \mathrm{cm}^{2}\right)$. Площадь светодиодов составляла $1 \mathrm{Mм}^{2}$.

В эксперименте измерялись зависимости внешнего квантового выхода от плотности протекающего тока (для ЭЛ) и интенсивности возбуждения (для ФЛ) в широком интервале температур $(T=10-300 \mathrm{~K})$. Максимальное значение квантового выхода, которое принималось за 1, наблюдалось при наиболее низких температурах.

При исследовании пользовались криогенной системой на базе криостата Janis CCS-300S/204 HT. Для плотности тока $j<3 \mathrm{~A} / \mathrm{cm}^{2}$ измерения проводились в статическом режиме при помощи источника-измерителя Keithley 2636. В импульсном режиме измерения проводились до $j=200 \mathrm{~A} / \mathrm{cm}^{2}$ на частотах 50 и 100 Гц (длительность $0.04 \cdot 10^{-3} \mathrm{c}$ ) при помощи осциллографа LeCroy104Xs. При исследовании КВ в режиме ФЛ пользовались YAG-лазером со средней мощностью $22.4 \cdot 10^{-3}$ В (длительность $10 \mathrm{Hc}$, частота $0.2-1$ кГц, длина волны 355 нм). Изменения интенсивности сфокусированного возбуждающего излучения добивались при помощи стеклянных ослабителей с покрытием ITO (indium-tin oxide). Спектры люминесценции и интегральная интенсивность излучения измерялись при помощи калиброванного кремниевого фотоприемника и оптоволоконного спектрометра USB 2000 (Ocean Optics).

Перегрев светодиодов исключался выбором высокой скважности измерений в импульсном режиме и использованием массивного медного держателя светодиодного кристалла (размеры $\sim 15 \times 10 \times 2$ мм), который прижимался винтом к холодному пальцу криостата. Об отсутствии перегрева судили по наличию коротковолнового сдвига люминесценции, характерного для структур $\mathrm{InGaN} / \mathrm{GaN}$ [1]. Об отсутствии эффекта стягивания тока судили по виду прямой ВАХ, на которой наблюдается резкий рост силы тока при увеличении напряжения для области спада КВ (области „efficiency droop“) [12,13]. Полагали, что стягивание тока должно приводить к обратному эффекту - увеличению сопротивления образцов.

\section{3. Результаты эксперимента}

Экспериментальные зависимости КВ $\eta$ от $T$, измеренные в различных режимах, представлены на рис. 1. При низких интенсивностях возбуждения в режиме ФЛ наблюдается спад квантового выхода при увеличении $T$, что согласуется с результатами других работ [1-3]. В этом случае температурная зависимость КВ обусловлена температурной зависимостью времен жизни излучательной рекомбинации и рекомбинации Шокли-Рида. В рамках $A B C$-модели квантовый выход для данного низкого уровня возбуждения определяется соотношением коэффициентов безызлучательной и излучательной рекомбинации.

Из рис. 1 следует, что при высоком уровне возбуждения для режима ФЛ наблюдается слабая температурная зависимость квантового выхода. Аналогичный результат был получен ранее в ряде работ для режимов ФЛ и ЭЛ $[1,3,15]$. Если учесть, что спад квантового выхода, который наблюдается в данной области, связан с ожепроцессом, то это указывает на слабую зависимость $C$ от $T$.

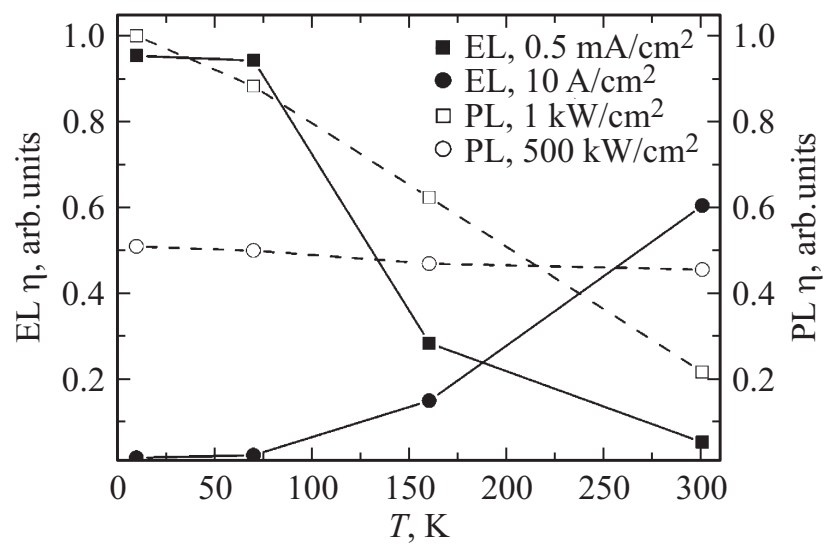

Рис. 1. Температурная зависимость квантового выхода $\eta$ светодиода с МКЯ InGaN/GaN при фото- (PL) и электролюминесценции (EL) для различных уровней возбуждения. 
Результаты исследования квантового выхода в режиме ЭЛ также качественно согласуются с результатами ряда работ [8-14]. В области невысокой плотности тока увеличение температуры приводит к уменьшению КВ, что также может быть обусловлено соотношением коэффициентов $A$ и $B$. В области высокой плотности тока имеет место сильная инверсия температурной зависимости КВ, по мере роста $T$ квантовый выход увеличивается более чем на порядок.

Стоит отметить, что для режима ЭЛ инверсия температурной зависимости квантового выхода в эксперименте наблюдается не всегда [20]. Так, в работах $[15,16]$ авторы наблюдали снижение КВ с увеличением температуры при высокой плотности тока, что в нашем случае аналогично режиму ФЛ. Возможное объяснение этого разногласия представлено в конце следующего раздела в рамках изложенной модели баллистической утечки.

\section{4. Обсуждение результатов}

\section{1. Низкотемпературные потери}

Качественное отличие температурной зависимости КВ в области высокого уровня инжекции для двух режимов говорит о том, что в режиме ЭЛ появляется дополнительный механизм потерь при низких температурах (дополнительный к потерям, связанным с ожерекомбинацией). При этом рассматриваемые потери не могут быть связаны с температурной зависимостью коэффициентов рекомбинации [20] или активацией рекомбинационных центров с меньшим временем жизни при увеличении концентрации носителей [22] (на это указывает сравнение результатов измерения квантового выхода при ФЛ и ЭЛ). Наиболее очевидным процессом, ответственным за потери, является утечка носителей заряда из активной области при высокой плотности тока. При этом наиболее вероятной является утечка электронов в $p$-область, так как они обладают меньшей эффективной массой и относительно однородно заполняют все квантовые ямы [24,25]. Дырки же в основном локализуются в области первых КЯ (со стороны $p$-слоя). Тем более это справедливо для низких температур, когда имеет место инжекция дырок из локализованных состояний акцептора $[12,26]$.

Использование представлений о квазиравновесном состоянии системы, положенных в основу диффузионнодрейфовой модели переноса заряда в структурах с КЯ $\mathrm{InGaN} / \mathrm{GaN}$ [27,28], не позволяет объяснить инверсию температурой зависимости КВ вследствие утечки. Главным образом это связано с экспоненциальным снижением вероятности термического выброса электронов из квантовых ям при уменьшении температуры. Косвенным подтверждением этому служат результаты работы [29], в которой проводился учет неоднородности состава слоев InGaN при трехмерном моделировании транспорта носителей в диффузионно-дрейфовом приближении. Было показано, что такая модель работает для температур, близких к комнатной; в области низких температур наблюдается расхождение расчетных и экспериментальных данных.

На наш взгляд, среди возможных механизмов низкотемпературной утечки следовало бы рассматривать туннелирование электронов в $р$-область по состояниям дефектов из активной области либо баллистический перенос электронов над КЯ. В настоящей работе рассматривается второй из упомянутых механизмов. Тем не менее публикаций по моделированию температурной инверсии КВ с учетом туннельной утечки в литературе нами также не обнаружено.

Баллистический перенос характеризуется временем релаксации носителей заряда; наиболее вероятным механизмом рассеяния энергии в $\mathrm{GaN}$ является взаимодействие с оптическими фононами [23]. При упругом рассеянии время релаксации, как и длина свободного пробега электронов, увеличивается при снижении температуры [30], что должно приводить к росту баллистической утечки. С другой стороны, известно, что сопротивление области МКЯ растет при снижении температуры [31,32]. Тогда падение напряжения на области МКЯ также будет увеличиваться при уменьшении $T$ для одинаковых плотностей тока. В итоге потенциальная энергия инжектированных из n-области электронов должна расти за счет приложенного к области МКЯ напряжения.

\section{2. Описание модели}

Для расчета зависимости КВ от $j$ и $T$ в настоящей работе используется ранее предложенная феноменологическая модель [13]. В данной модели область МКЯ разбивается на две части: область протекания тока, ограниченного пространственным зарядом (SCLC); область пространственного заряда электронно-дырочного перехода (SCR). Для описания модели на рис. 2 схематично представлена энергетическая диаграмма активной области светодиода с 7 КЯ в равновесии (a) и при прямом смещении $V(b)$. Предполагается, что снижение температуры приводит к увеличению роли туннельной составляющей проводимости МКЯ, когда носители из одной квантовой ямы переходят в другую за счет прыжкового транспорта (туннелирование по дефектам). В силу того что в запрещенной зоне барьеров GaN существует хвост плотности состояний, преимущественно заряд переносится на некотором транспортном уровне, положение которого зависит от температуры. В этом смысле заимствована модель переноса заряда, предложенная ранее для аморфных полупроводников [33]. Учитывается также, что локализованные состояния квантовых ям могут выступать в роли мелких электронных ловушек. Ранее нами было показано, что использование такого подхода позволяет описать экспериментальные ВАХ в области низких температур до пороговой плотности тока, соответствующей началу участка спада КВ [13]. В этом случае плотность тока, ограниченного пространственным зарядом, захваченным на мелкие ловушки, 


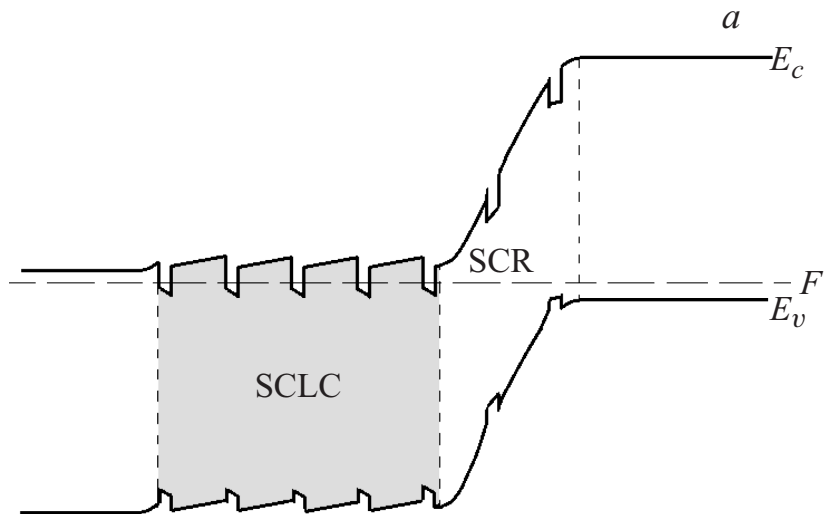

$b$

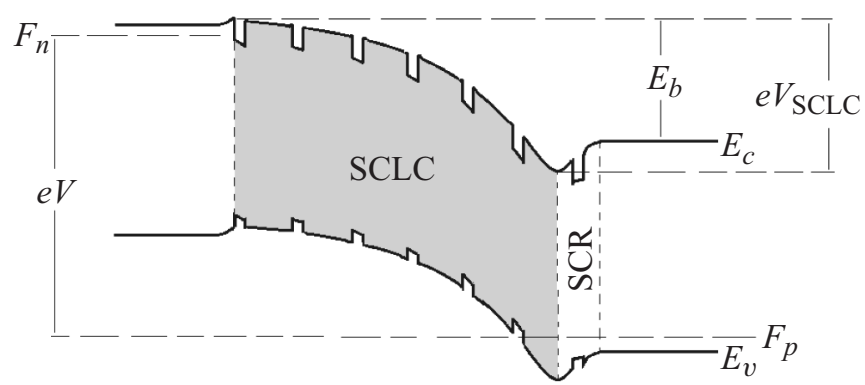

Рис. 2. Энергетическая диаграмма светодиода с МКЯ $\mathrm{InGaN} / \mathrm{GaN}$ в равновесии $(a)$ и при прямом смещении $V(b)$. $E_{c}, E_{v}$ - края зоны проводимости и валентной зоны, $F-$ уровень Ферми, $F_{n}$ и $F_{p}-$ квазиуровни Ферми для электронов и дырок.

подчиняется выражению [13]

$$
j_{t}=[r(T)]^{-2} V_{\mathrm{SCLC}}^{2}
$$

где $V_{\mathrm{SCLC}}-$ напряжение на области накопления заряда в МКЯ, $r(T)$ - эмпирический коэффициент, который находится из экспериментальных ВАХ.

Здесь следует уточнить, что механизм ограничения тока в области МКЯ может иметь иную природу. Тем не менее эмпирическая формула (1) остается справедливой и может быть использована в моделировании.

При достижении некоторого значения прямого напряжения на светодиоде инжектированные из $n$-области электроны приобретают дополнительную энергию $E_{b}$, что приводит к росту баллистической составляющей проводимости через всю область МКЯ (рис. $2, b$ ). Плотность баллистического тока утечки рассчитывается согласно следующему выражению [13]:

$$
\begin{aligned}
j_{b}\left(V_{b}\right)= & e \int_{0}^{\infty} f(E, T) N(E, T) \exp \left[-\frac{W}{\tau_{s c} v\left(E+e V_{b}, T\right)}\right] \\
& \times v\left(E+e V_{b}, T\right) d E,
\end{aligned}
$$

где $e$ - заряд электрона, $V_{b}=E_{b} / e, W-$ толщина активной области светодиода, $E$ - энергия электронов относительно дна зоны проводимости, $f-$ функция Ферми-Дирака, $N$ - плотность квантовых состояний в зоне проводимости, $v-$ скорость электронов, $\tau_{s c}-$ время релаксации для электронов.

Для учета различных механизмов рекомбинации в настоящей работе плотность рекомбинационного тока $\left(j_{R}\right)$ рассчитывается согласно выражению

$$
\begin{array}{r}
j_{R}\left(V_{p-n}\right)=j_{\text {nrad }}+j_{\text {rad }}=e d\left[A n_{i} \exp \left(\frac{e V_{p-n}}{2 k T}\right)\right. \\
\left.+C n_{i}^{3} \exp \left(\frac{3 e V_{p-n}}{2 k T}\right)+B n_{i}^{2} \exp \left(\frac{e V_{p-n}}{2 k T}\right)\right],
\end{array}
$$

где $d-$ ширина квантовой ямы (предполагается, что при низких температурах рекомбинация носителей происходит только в первой со стороны р-области квантовой яме $[12,13]), n_{i}$ - концентрация собственных носителей, $V_{p-n}$ - напряжение, приложенное к области пространственного заряда электронно-дырочного перехода.

Аналитическое выражение (3) является упрощенным и получено из общего определения рекомбинационного тока без учета диффузионно-дрейфового перераспределения носителей в пределах активной области (ток ограничивается только рекомбинацией в одной КЯ). Схема вывода плотности рекомбинационного тока для линейного и квадратичного случев представлена в [34], аналогично выводится зависимость $j=j\left(V_{p-n}\right)$ для кубической рекомбинации. Использование выражения (3) позволяет учесть выводы $A B C$-модели при расчете зависимости КВ от $j$ и $T$.

Таким образом, рассматриваемая модель базируется на решении системы уравнений (1)-(3) для эквивалентной схемы активной области светодиода, представленной на рис. 3. Для моделирования зависимости КВ от $j$ и $T$ используется следующее выражение:

$$
\eta(j)=j_{\text {rad }} / j_{\text {total }},
$$

где $j_{\text {total }}-$ плотность полного тока через всю структуру, являющаяся суммой выражений (1) и (2). Напряжение на МКЯ рассчитывается согласно выражению $V=V_{\mathrm{SCLC}}+V_{p-n}$.

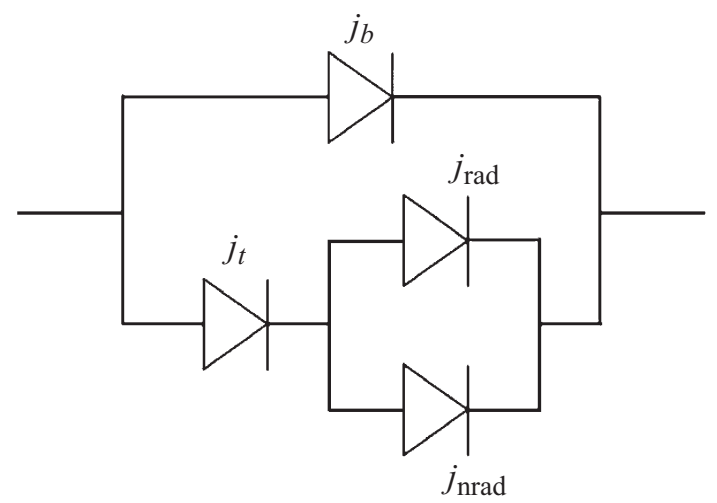

Рис. 3. Эквивалентная схема активной области светодиода с МКЯ InGaN/GaN, используемая в моделировании. 


\section{3. Результаты моделирования}

Описанная модель содержит много неизвестных параметров, главным образом $A, B, C$ и $\tau_{s c}$. Данные параметры должны зависеть от температуры и в некоторой степени определяться конструкцией и технологией изготовления экспериментальных образцов. Измерение их температурных зависимостей затруднительно, и поэтому на данном этапе исследований представляется возможным лишь качественный анализ с использованием литературных сведений о значениях величин $A, B$, С и $\tau_{s c}$.

В качестве не зависящих от температуры в расчете использовались следующие параметры: $C=10^{-30} \mathrm{~cm}^{6} / \mathrm{c} \quad[19], \quad \tau_{s c}=9 \cdot 10^{-15} \mathrm{c} \quad[23], d=2 \mathrm{HM}$, $W=100$ нм. Хотя коэффициент оже-рекомбинации должен расти с повышением температуры за счет роста тепловой скорости носителей, согласно экспериментальным данным зависимость $C(T)$ является слабой, о чем упоминалось в разд. 3. С другой стороны, параметр $\tau_{s c}$ может увеличиваться при снижении температуры [30]. Тем не менее использование точных зависимостей $C$ и $\tau_{s c}$ от $T$ не должно повлиять на качественный вид зависимости квантового выхода от $T$ и $j$, а увеличение $\tau_{s c}$ при снижении $T$ должно приводить к усилению процесса утечки электронов при низких температурах.

Значения параметров, зависящих от $T$, представлены в таблице. Значения $r(T)$ получены путем аппроксимации экспериментальной ВАХ светодиода с учетом выражения (1) по методике, изложенной в $[13,32]$. Значения коэффициентов $A$ и $B$ для комнатной температуры взяты из работы [19]. Температурные зависимости коэффициентов рекомбинации выбраны из общих представлений о том, что время жизни относительно безызлучательной рекомбинации с понижением температуры увеличивается, а время жизни относительно излучательной рекомбинации уменьшается [21]. Порядок изменения $A$ и $B$ при понижении температуры выбирался согласно данным работы [22], это позволило добиться качественного согласия расчетных и экспериментальных зависимостей $\eta(T, j)$ для области низких плотностей тока.

Результаты моделирования представлены на рис. 4 и 5 . Из рис. 4 следует, что увеличение температуры приводит к сдвигу зависимости $\eta(j)$ в область высоких плотностей тока, что качественно согласуется с экспериментальными данными различных работ $[8,9,11-14]$. В результате этого в области высоких плотностей тока появляется инверсия температурной зависимости квантового выхода (рис. 5).

Значения параметров, использованных в моделировании зависимости квантового выхода от $T$ и $j$

\begin{tabular}{c|c|c|c|c|c}
\hline $\begin{array}{c}\text { Tемпера- } \\
\text { тура, K }\end{array}$ & 80 & 160 & 220 & 300 & 360 \\
\hline$A, \mathrm{c}^{-1}$ & $0.3 \cdot 10^{6}$ & $2 \cdot 10^{6}$ & $8 \cdot 10^{6}$ & $1.5 \cdot 10^{7}$ & $2 \cdot 10^{7}$ \\
$B, \mathrm{~cm}^{3} \cdot \mathrm{c}^{-1}$ & $5 \cdot 10^{-10}$ & $2 \cdot 10^{-10}$ & $4 \cdot 10^{-11}$ & $1.5 \cdot 10^{-11}$ & $1 \cdot 10^{-11}$ \\
$r, \mathrm{~B} / \mathrm{A}^{1 / 2}$ & 106 & 64 & 5 & 1.5 & 0.5
\end{tabular}

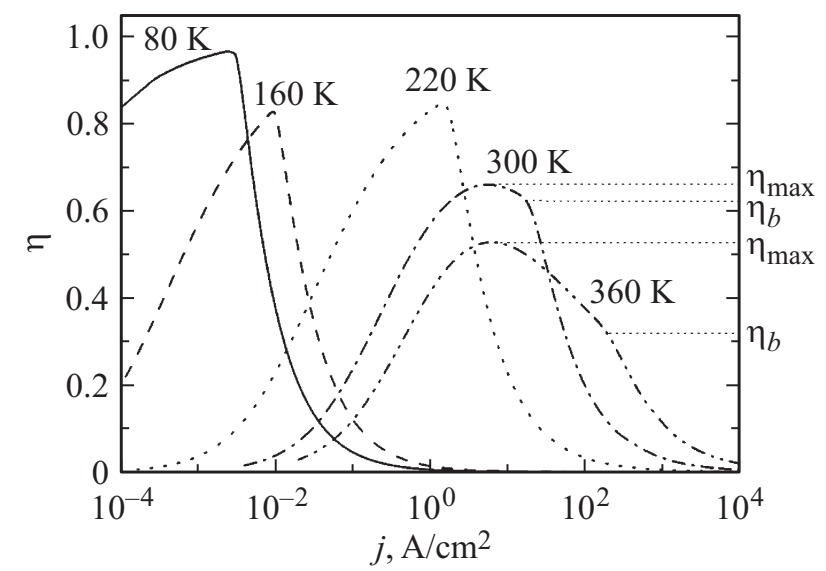

Рис. 4. Зависимости квантового выхода электролюминесценции светодиода с МКЯ $\mathrm{InGaN} / \mathrm{GaN}$ от плотности тока, рассчитанные для различных температур (коэффициент вывода излучения принимался равным 1).

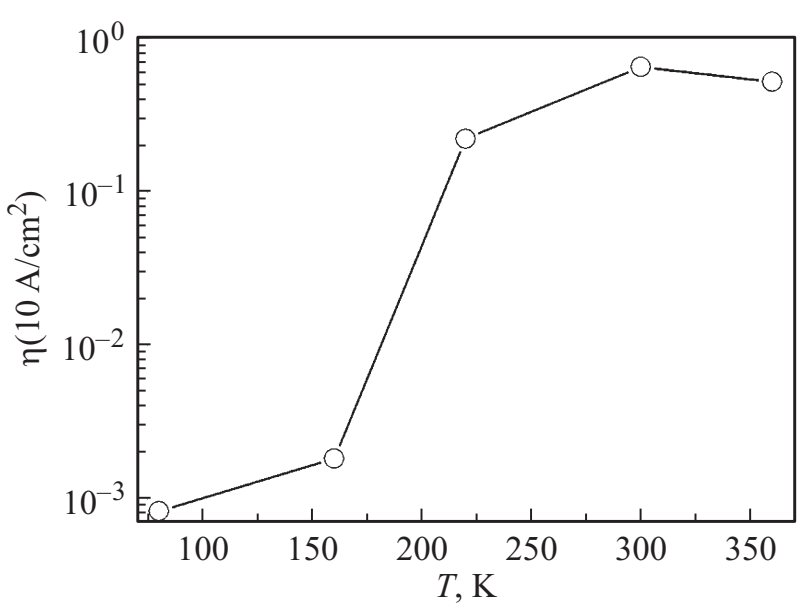

Рис. 5. Рассчитанная температурная зависимость квантового выхода электролюминесценции светодиода с МКЯ InGaN/GaN для плотности тока $j=10 \mathrm{~A} / \mathrm{cm}^{2}$.

Как показано на рис. 4, зависимость КВ от $j$ в области спада квантового выхода качественно изменяется при повышении $T$ от 220 до $300 \mathrm{~K}$. При низких температурах $(T \leq 220 \mathrm{~K})$ КВ резко снижается с увеличением $j$ по причине роста баллистической утечки, или, что то же самое, увеличения отношения $j_{b} / j_{R}$. При высоких температурах $(T \geq 300 \mathrm{~K})$ область спада КВ можно разбить на две части. Первая соответствует относительно слабому уменьшению КВ при увеличении $j$ (интервал $\eta_{b}<\eta<\eta_{\max }$, изображенный на рис. 4). Анализ модели показывает, что данная область связана с влиянием процесса оже-рекомбинации. Вторая часть характеризуется резким снижением КВ (интервал $0<\eta<\eta_{b}$, изображенный на рис. 4). В данном случае природа снижения КВ такая же, как при $T \leq 220 \mathrm{~K}$.

Таким образом, из результатов моделирования следует, что оже-рекомбинация имеет наибольшее влияние на эффект спада квантового выхода при увеличении $j$ для 
температур, близких комнатной (и выше). При более низких температурах наиболее существенное влияние на спад КВ оказывает баллистическая утечка электронов в p-область.

Дополнительные расчеты показали, что роль баллистической утечки снижается при уменьшении падения напряжения на области МКЯ. Формально это означает, что чем меньше параметр $r$, тем при бо́льших значениях $j$ начинается баллистическая утечка. В пределе, при $r=0$, описанная модель полностью переходит в $A B C$-модель, когда отсутствует инверсия температурной зависимости КВ в области высоких $j$. При этом на ВАХ не наблюдается ограничения тока, кроме омических потерь на последовательном сопротивлении контактов и пассивных областях светодиода. С этих позиций становится очевидным объяснение результатов ряда работ, в которых экспериментальные зависимости $\eta(T, j)$ описываются $A B C$-моделью. Вероятно, $A B C$-модель применима для светодиодов, активная область которых содержит малое количество КЯ, сверхрешетку (набор КЯ, разделенных барьерами с малой толщиной) либо не содержит дополнительных буферных слоев со стороны n-слоя, приводящих к ограничению тока при низких температурах. В таких светодиодах сопротивление активной области мало́ в силу однородного растекания дырок и электронов по всем слоям структуры. Однако анализ с этих позиций затруднителен для уже имеющихся в литературе данных. Это связано с тем, что большая часть исследований проводилась ранее на коммерческих светодиодах $[8,9,11,15,16]$, особенности строения активной области которых детально не описываются.

\section{5. Заключение}

В работе рассмотрены различные случаи экспериментального наблюдения инверсии температурной зависимости квантового выхода в режимах фото- и электролюминесценции. Наибольший практический интерес вызывает режим электролюминесценции в области высокой плотности тока $\left(j>1-10 \mathrm{~A} / \mathrm{cm}^{2}\right)$. Показано, что при высокой плотности тока может наблюдаться инверсия температурной зависимости квантового выхода, когда квантовый выход резко возрастает при увеличении температуры. Для объяснения данного эффекта использована модель баллистической утечки, учитывающая увеличение сопротивления области множественных квантовых ям при снижении температуры. Анализ результатов моделирования показал, что в области низких температур спад квантового выхода обусловлен баллистической утечкой электронов в $p$-область, в то время как при температурах выше комнатной спад также связан и с оже-рекомбинацией. Вследствие этого наблюдается рост квантового выхода с увеличением температуры при $T<220-300 \mathrm{~K}$. Рассмотренная модель позволяет качественно объяснить экспериментальные результаты, а ее базовые предположения могут быть использованы при построении более строгой теории транспорта носителей заряда в структурах с множественными квантовыми ямами $\mathrm{InGaN} / \mathrm{GaN}$.

Работа выполнена при финансовой поддержке Министерства образования и науки России (гос. задание № 3.1206.2014) и Программы повышения международной конкурентоспособности Томского государственного университета на 2013-2020 гг.

\section{Список литературы}

[1] S. Watanabe, N. Yamada, M. Nagashima, Y. Ueki, C. Sasaki, Y. Yamada, T. Taguchi, K. Tadatomo, H. Okagawa, H. Kudo. Appl. Phys. Lett., 83 (24), 4906 (2003).

[2] A. Sasaki, S. Shibakawa, Y. Kawakami, K. Nishizuka, Y. Narukawa, T. Mukai. Jpn. J. Appl. Phys., 45 (11), 8719 (2006).

[3] T. Kohno, Y. Sudo, M. Yamauchi, K. Mitsui, H. Kudo, H. Okagawa, Y. Yamada. Jpn. J. Appl. Phys., 51, 072102 (2012).

[4] Y.C. Shen, G.O. Mueller, S. Watanabe, N.F. Gardner, A. Munkholm, M.R. Krames. Appl. Phys. Lett., 91, 141101 (2007).

[5] J.H. Chen, Z.C. Feng, J.C. Wang, H.L. Tsai, J.R. Yang, A. Parekh, E. Armour, P. Faniano. J. Cryst. Growth, 287, 354 (2006).

[6] И.А. Прудаев, И.С. Романов, В.В. Копьев, С.Б. Ширапов, О.П. Толбанов, С.С. Хлудков. Изв. вузов. Физика, 56 (7), 30 (2013).

[7] J. Ma, X. Ji, G. Wang, X. Wei, H. Lu, X. Yi, R. Duan, J. Wang, Y. Zeng, J. Li, F. Yang, C. Wang, G. Zou. Appl. Phys. Lett., 101, 131101 (2012).

[8] Н.И. Бочкарева, Е.A. Zhirnov, А.А. Ефремов, Е.Т. Ребане, Р.И. Горбунов, Ю.Г. Шретер. ФТП, 39 (5), 627 (2005).

[9] D.S. Meyaard, G.-B. Lin, Q. Shan, J. Cho, E.F. Schubert, H. Shim, M.-H. Kim, C. Sone. Appl. Phys. Lett., 99, 251115 (2011).

[10] А.С. Павлюченко, И.В. Рожанский, Д.А. Закгейм. ФТП, 43 (10), 1391 (2009).

[11] K. Fudjiwara, H. Jimi, K. Kaneda. Phys. Status Solidi C, 6 (S2), S814 (2009).

[12] И.А. Прудаев, И.Ю. Голыгин, С.Б. Ширапов, И.С. Романов, С.С. Хлудков, О.П. Толбанов. ФТП, 47 (10), 1391 (2013).

[13] I. Prudaev, O. Tolbanov, S. Khludkov. Phys. Status Solidi A, 212 (5), 930 (2015).

[14] C.H. Wang, J.R. Chen, C.H. Chiu, H.C. Kuo, Y.-L. Li, T.C. Lu, S.C. Wang. IEEE Photon. Tech. Lett., 22 (4), 236 (2010).

[15] I.E. Titkov, S.Yu. Karpov, A. Yadav, V.L. Zerova, M. Zulonas, B. Galler, M. Strassburg, I. Pietzonka, H.-J. Lugauer, E.U. Rafailov. IEEE J. Quant. Electron., 50 (11), 911 (2014).

[16] A. Laubsch, M. Sabathil, W. Bergbauer, M. Strassburg, H. Lugauer, M. Peter, S. Lutgen, N. Linder, K. Streubel, J. Hader, J.V. Moloney, B. Pasenow, S.W. Koch. Phys. Status Solidi C, 6 (S2), S913 (2009).

[17] В.С. Сизов, В.В. Неплох, А.Ф. Цацульников, А.В. Сахаров, В.В. Лундин, Е.Е. Заварин, А.Е. Николаев, А.М. Минтаиров, J.L. Merz. ФТП, 44 (12), 1615 (2010).

[18] M.F. Schubert, J. Xu, Q. Dai, F.W. Mont, J.K. Kim, E.F. Schubert. Appl. Phys. Lett., 94, 081114 (2009).

[19] J. Piprek. Phys. Status Solidi A, 207 (10), 2217 (2010).

[20] S. Yu. Karpov. Opt. Quantum Electron., 47 (6), 1293 (2015). 
[21] Ф.Е. Шуберт. Светодиоды, пер. под ред. А.Э. Юновича (М., физматлит, 2008) гл. 2, с. 60.

[22] J. Hader, J.V. Moloney, S.W. Koch. Appl. Phys. Lett., 99, 181127 (2011).

[23] X. Ni, X. Li, J. Lee, S. Liu, V. Avrutin, A. Matulionis, U. Ozgur, H. Morkoc. Superlat. Microstruct., 48, 133 (2010).

[24] А.В. Сахаров, В.В. Лундин, Е.Е. Заварин, М.А. Синицын, А.Е. Николаев, С.О. Усов, В.С. Сизов, Г .А. Михайловский, Н.А. Черкашин, М. Hytch, F. Нuе, Е.В. Яковлев, А.В. Лобанова, А.Ф. Цацульников. ФТП, 43 (6), 841 (2009).

[25] D.A. Zakheim, A.S. Pavluchenko, D.A. Bauman, K.A. Bulashevich, O.V. Khokhlev, S.Yu. Karpov. Phys. Status Solidi A, 209 (3), 456 (2012).

[26] D. Yang, L. Wang, W.-B. Lv, Z.-B. Hao, Y. Luo. Superlat. Microstruct., 82, 26 (2015).

[27] K.A. Bulashevich, V.F. Mymrin, S.Yu. Karpov, I.A. Zhmakin, A.I. Zhmakin. J. Comp. Phys., 213, 214 (2006).

[28] S.Yu. Karpov. Proc. SPIE Gallium Nitride Materials and Devices VI (San Francisco, USA, 2011) v. 7939, p. 79391C.

[29] D.A. Browne, B. Mazumder, Y.-R. Wu, J.S. Speck. J. Appl. Phys., 117, 185703 (2015).

[30] А.И. Ансельм. Введение в физику полупроводников (М., Наука, 1978) гл. 8, п. 6, с. 484.

[31] И.А. Прудаев, И.В. Ивонин, О.П. Толбанов. Изв. вузов. Физика, 54 (12), 66 (2011).

[32] И.А. Прудаев, Ю.Л. Зубрилкина, А.А. Бактыбаев, И.С. Романов. Изв. вузов. Физика, 57 (9), 86 (2014).

[33] Don Monroe. Phys. Rev. Lett., 54, 146 (1985).

[34] П.Г. Елисеев. Введение в физику инжекционных лазеров (М., Наука, 1983) гл. 6, с. 186.

Редактор Л.В. Шаронова

\section{The influence of ballistic overflow on temperature dependence of quantum efficiency of InGaN/GaN multiple quantum well light-emitting diodes}

\section{I.A. Prudaev, V.V. Kopyev, I.S. Romanov, V.L. Oleynik}

Tomsk State University, 634050 Tomsk, Russia

Abstract In this work for $\mathrm{InGaN} / \mathrm{GaN}$ multiple quantum well light-emitting diodes the dependences of quantum efficiency on temperature and excitation level are investigated. The experiment was conducted for two regimes of luminescence excitation. Comparison of results obtained under photo- and electroluminescence showed that at the high current densities the additional lowtemperature energy loss appears (additional to Auger-recombination loss). It leads to temperature inversion of quantum efficiency at the temperatures less than $220-300 \mathrm{~K}$. The analysis of the results has shown that the loss is associated with electron leakage from the active region of a light-emitting diode. To explain the experimental data the model of ballistic overflow was used. The results of simulation qualitatively agrees with experimental dependences of quantum efficiency on temperature and current density. 